www.nature.com/pj

\title{
New directions in the design of phenylboronate- functionalized polymers for diagnostic and therapeutic applications
}

\begin{abstract}
Akira Matsumoto $^{1}$, Kazunori Kataoka ${ }^{2}$ and Yuji Miyahara ${ }^{1}$
Phenylboronic acid (PBA) derivatives, which are often termed 'boronolectins,' are regarded as a synthetic mimic of lectins because of their ability to interact with various carbohydrates. This unique chemistry has already borne fruit as the molecular bases for glucose sensors and some bio-separation applications. This focus review highlights some of the emerging PBA-based research directions toward more versatile diagnostic and therapeutic targets, which the authors are currently pursuing. The topics reviewed include PBA-mediated targeting to sialic acid (SA) as a methodology relevant to tumor diagnosis and treatment. Other drug delivery systems, including those for siRNA and insulin, in which PBA has a unique role in physicochemical signal transduction, will also be summarized.
\end{abstract}

Polymer Journal (2014) 46, 483-491; doi:10.1038/pj.2014.42; published online 11 June 2014

Keywords: diagnosis; drug delivery system; insulin; phenylboronic acid; sialic acid; siRNA

\section{INTRODUCTION}

Phenylboronic acid (PBA) derivatives readily complex with 1,2- and 1,3-cis-diol compounds, including those found in carbohydrates, through reversible boronate ester formation in an aqueous solution (Scheme 1). ${ }^{1-4}$ Because of this property, PBA derivatives, which are often termed 'boronolectins,' can be regarded as synthetic, remarkably stable alternatives to lectins (carbohydrate-binding proteins) whose binding specificity can be chemically tailored. Lorand and Edwards were the first to quantitatively reveal this fascinating property of PBA. Since the late 1960s, PBA compounds have been extensively studied as ligands for affinity chromatography for the purification of polyol compounds, including ribonucleic acid (RNA). ${ }^{5-9}$ Optical and electrical chemosensing applications, such as those focusing on glucose detection, were the next research trend to emerge, ${ }^{10-14}$ and the first concept appeared in the early 1990s. ${ }^{10-12}$ Boronic acid compounds are compatible with human physiology, as exemplified by the fact that some of them have been used as chemotherapeutic agents and in other therapies. ${ }^{15-17}$ Meanwhile, PBA interacts with a variety of biological membranes, including those of cells, viruses, bacteria and fungi, through the membrane-constituting carbohydrate moieties. ${ }^{18-22}$ To illustrate this point, some PBA-functionalized synthetic polymers can specifically bind to glycoproteins of lymphocytes and induce their enhanced proliferation, a feature truly mimicking that of lectins that is relevant for immunotherapy. ${ }^{22}$ These interactions provide strong rationale for the design of
PBA-based synthetic ligands and cell-targeting strategies for drug delivery. An attractive characteristic for drug delivery applications is that PBA is able to undergo a marked inversion in its state of hydrophobicity, depending on the degree of acid disassociation; ${ }^{23}$ it is strongly hydrophobic when uncharged, but it becomes hydrophilic when it is anionically charged at $\mathrm{pH}$ values above its $\mathrm{pKa}$. Because the PBA-diols interaction is a dynamic (equilibrium) event determined by the respective concentrations, these features lead to an ability to fine-tune or switch the stability of the complex, offering a new strategy for environmental sensitivity. ${ }^{24,25}$

In this focus review, we aim to summarize some new aspects of PBA (polymer)-based research directed toward diagnostic and therapeutic applications, focusing on our own contributions, unless otherwise stated. The topic will include PBA-mediated targeting to sialic acid (SA), an anionic monosaccharide that frequently occurs at the termini of glycan chains, as a relevant technique for tumor diagnosis and treatment. Other unique PBA-based insulin and siRNA delivery systems will also be described.

\section{SIALIC ACID RECOGNITION AS A NEW TOOL FOR DIAGNOSTIC AND THERAPEUTIC APPLICATIONS}

Glycosylations, or the alternations of glycoforms, are dynamic and stage-specific processes that occur during numerous normal and pathological events, including development, differentiation, infection, genetic disorders and cancers. ${ }^{26-28}$ SAs are the most abundant

${ }^{1}$ Institute of Biomaterials and Bioengineering, Tokyo Medical and Dental University, Tokyo, Japan and ${ }^{2}$ Department of Materials Engineering, Graduate School of Engineering, The University of Tokyo, Tokyo, Japan

Correspondence: Professor A Matsumoto, Institute of Biomaterials and Bioengineering, Tokyo Medical and Dental University, 2-3-10 Kanda-surugadai, Chiyoda-ku, Tokyo, 101-0062, Japan.

E-mail: matsumoto.bsr@tmd.ac.jp

Received 20 March 2014; revised 10 April 2014; accepted 11 April 2014; published online 11 June 2014 

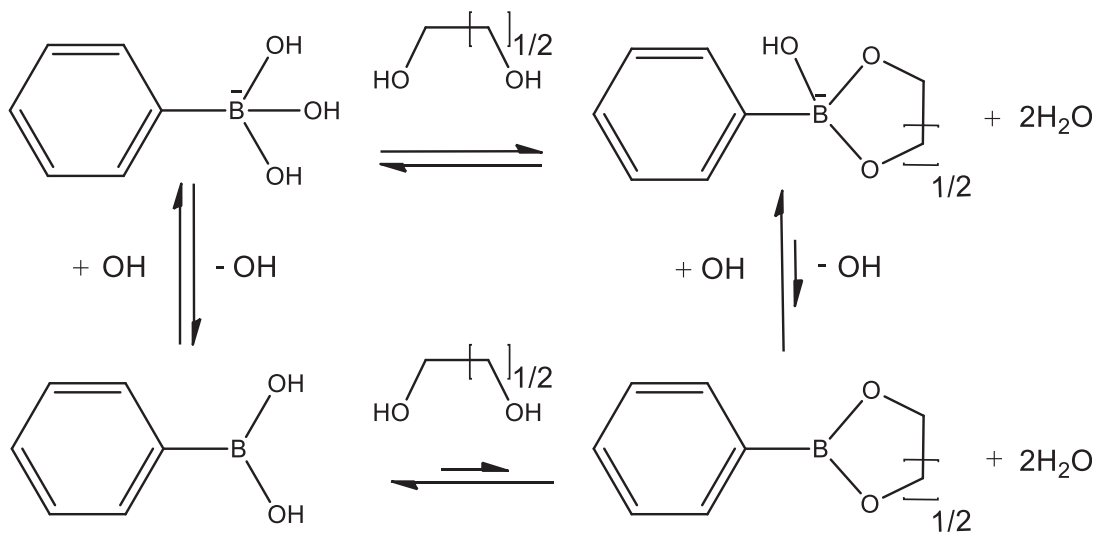

Scheme 1 Reversible boronate ester formation in aqueous solution.
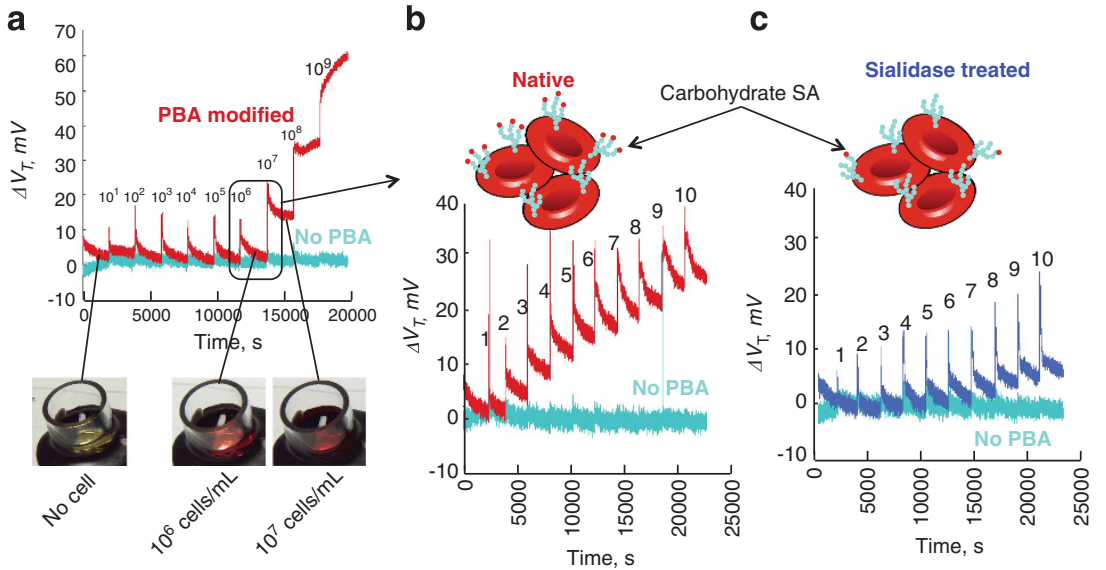

Figure 1 (a) Potentiometric responses upon adding rabbit erythrocytes to a PBA-modified field effect transistor (FET). The concentrations are indicated in numbers (cells $\mathrm{ml}^{-1}$ ) at the time points of each addition. (b, c) Potentiometric responses upon increasing the erythrocyte concentration in steps of $10^{6} \mathrm{cells} \mathrm{ml}^{-1}$, investigated for native and enzymatically treated cells. Numbers denoted in the graph indicate the concentrations of the cell suspensions (million cells $\mathrm{ml}^{-1}$ ) and the time points of each addition. Reproduced, with permission, from Matsumoto et al. ${ }^{36}$

terminal residues of carbohydrate chains. The increased expression of sialylated antigens on cells is a hallmark of cancer progression and is an indicator of poor prognosis in patients with lung, breast, colon, prostate, bladder and stomach cancer. ${ }^{29-31}$ Moreover, hypoxic regions of solid tumors have shown increased levels of sialylated antigens, ${ }^{32}$ and the overexpression of sialylated glycans is important for the formation of metastases. ${ }^{33}$ Therefore, techniques to determine SA could be useful for both diagnostic and therapeutic applications related to those diseases. The gold standard methodology for the determination of SA is to exploit fluorophore-labeled reagents, often in combination with SA-specific lectins. However, these methods involve cell-lethal, costly and time-consuming procedures, as SA residues must be either enzymatically or acid-catalytically cleaved from the glycan chains before they can be quantified. In this regard, PBA-SA interactions provide an attractive alternative option.

Although most sugars favor tetrahedral boronate in complexation, PBA-SA binding is also possible with trigonal boronic acids. This anomalous binding behavior has been correlated with the special binding modalities taken by the PBA-SA complexes, in which multiple metastable binding sites and intramolecular stabilization via $\mathrm{B}-\mathrm{N}$ or $\mathrm{B}-\mathrm{O}$ interactions are suggested to have adjunctive roles. ${ }^{34,35}$ An important implication of the SA-binding phenomenon is that a PBA derivative with properly modulated $\mathrm{pKa}$ could provide a molecular basis for the selective recognition of SA among other glycan constituent saccharides.

Our recent work has described the PBA-based potentiometric detection of SA, establishing a new platform for non-invasive and label-free cytology. ${ }^{36,37}$ A PBA-modified self-assembled monolayer was immobilized on the surface of a gold electrode, which was used as an extended gate of a field effect transistor. ${ }^{38}$ The specific binding between negatively charged SA and PBA produced a change in the surface potential. The SA-specific signal was first confirmed at physiological $\mathrm{pH}$; the electrode was no longer specific to SA under alkaline conditions, consistent with the binding properties of PBA described above. It should be noted that, as a nature of the field effect, charge detection is inherently permitted only within a short distance corresponding to the electrical double layer, or the Debye length, which is no greater than a few nanometers, even under minimized ionic strength conditions. However, this requirement is compatible with the detection of cell surface SA moieties, which generally dominate the termini of the glycan chains. Tumor or metastasisassociated overexpression of SA is usually found in the form of polysialylation. Such a unit-sequential arrangement of the target SA (homopolymer of SA) present on the glycan chain termini may also aid in achieving a precise reflection of the altered level of SA expression. Moreover, the short detection length of the technique can beneficially restrict the charge detection to the molecules that are 


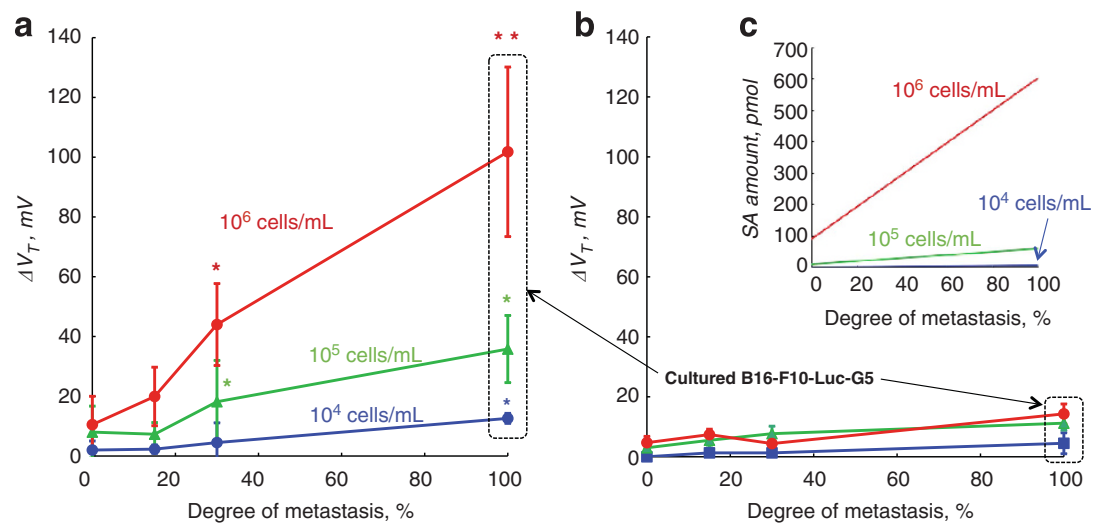

Figure 2 Equilibrium potentiometric changes found by adding cell suspensions to (a) PBA-modified and (b) non-modified gold electrodes as a function of the degree of metastasis for various cell concentrations. Data sets for the 100\% tumoral fraction (dashed boxes) were obtained from cultured B16-F10-Luc-G5 (no pneumocytes), whereas those for $0 \%$ were obtained from healthy pneumocytes. Data are expressed as averages \pm s.d. values $\left(n=6,{ }^{*} P<0.05\right.$ and $* * P<0.01$, respectively, versus $0 \%$ metastasis for the same cell concentrations). Reproduced, with permission, from Matsumoto et al. ${ }^{37}$ (c) Amount of SA present per electrode well under equivalent conditions to those investigated in (a) and (b), as determined by an enzymatic method with a commercial kit (SIALICQ, Sigma).

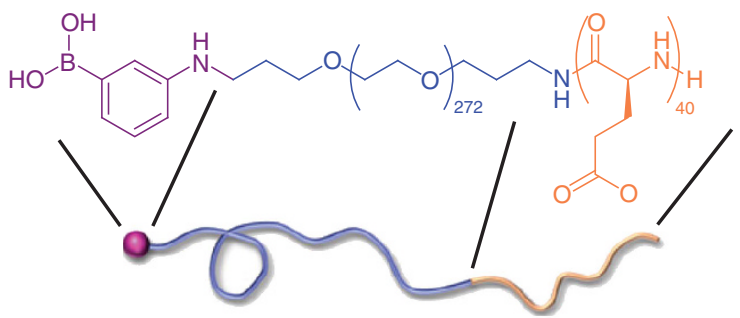

3-Aminophenylboronic acid-PEG-b-Poly(L-glutamic acid) (PBA-PEG-PLGA)

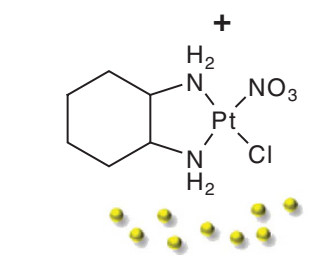

(1,2-diaminocyclohexane)platinum(II) (DACHPt) nitrate chloride

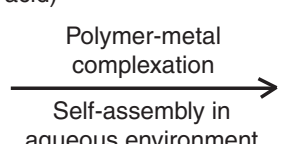
aqueous environment

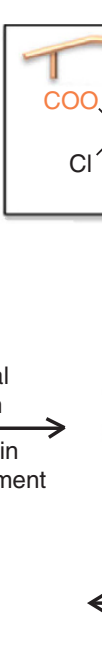

PBA-installed DACHPt-loaded micelles (PBA-DACHPt/m)

Figure 3 Preparation of PBA-installed DACHPt-loaded micelles by self-assembly through polymer-metal complex formation between DACHPt and PBA-PEG- $b$-PLGA in distilled water. PBA moieties on the surface of the micelles can bind to SA. Reproduced, with permission, from Deshayes et al. ${ }^{47}$

truly (covalently) bound to the electrode surface within the Debye length scale vicinity (that is, PBA-bound SA) and exclude other charges bound through non-specific or non-covalent interactions. In this context, utilizing small molecule ligands, such as PBA, rather than protein-based ones, such as antibodies and lectins, whose typical sizes are much larger than the Debye length, is a rational approach to designing field effect transistor -based sensors.

To demonstrate the ability of the electrode to capture SA moieties on cell membranes, erythrocytes were first investigated, as a decline in the SA content of erythrocytes has been implicated in diabetes mellitus. ${ }^{39-41}$ As shown in Figure 1, altered SA expression could be assessed in real-time simply by placing suspensions with a known number of living cells onto the electrode. ${ }^{36}$ The electrode was further applied to assess the malignancy or the degree of metastasis of tumor tissues. $^{37}$ For this purpose, metastatic murine melanoma cells expressing luciferase (B16-F10-Luc-G5) were utilized for their ability to specifically metastasize to the lungs of healthy mice after intravenous injection. ${ }^{42-44}$ This system enabled the facile preparation of tumoral lung specimens with various degrees of metastases, depending on the incubation times after each injection. Figure 2 summarizes the potential shift as a function of the degree of metastasis for various cell concentrations, wherein data sets for cultured melanoma (as a model for 100\% metastasis), healthy pneumocytes ( $0 \%$ metastasis) and the control series using an electrode without PBA modification are also indicated (Figure $2 b$ ). These results demonstrate that an advancement of metastasis in the living tissue can be detected using the PBA-modified electrode. This technique may serve as a quantitative adjunct to histological evaluations of tumor malignancy and metastatic potential during intra- or post-operative diagnoses.

The PBA-SA interaction described above has also been exploited to label sialylated epitopes in vitro and in vivo. ${ }^{45,46}$ In addition, the fact that elevated sialylation is a common signature of tumor malignancy suggests a new strategy for delivering bioactive or -inactive substances 

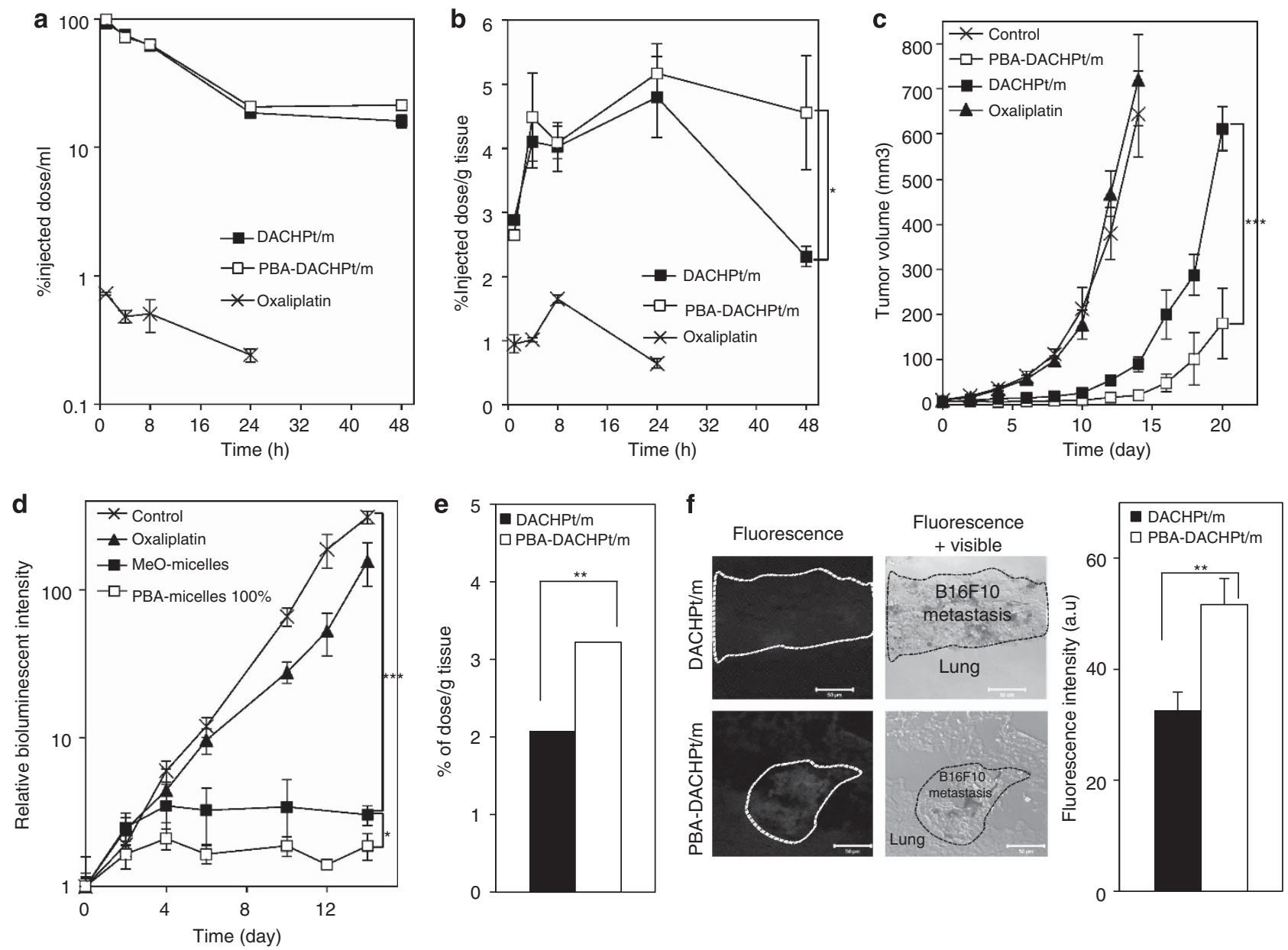

Figure 4 In vivo properties of PBA-DACHPt $/ \mathrm{m}$. (a) Plasma clearance and (b) tumor accumulation of DACHPt $/ \mathrm{m}$ and PBA-DACHPt/m in mice bearing a B16F10 tumor model. Data are means \pm s.e.m.; $n=5 ;{ }^{*} P<0.05$. (c) Antitumor activity against orthotopic B16F10 tumors after treatment with oxaliplatin $\left(8 \mathrm{mg} \mathrm{kg}^{-1}\right)$, DACHPt $/ \mathrm{m}$ or PBA-DACHPt $/ \mathrm{m}\left(3 \mathrm{mg} \mathrm{kg}^{-1}\right)$ injected on days 0,2 and 4 . Data are expressed as averages \pm s.e.m.; $n=5 ; * * * P<0.001$. (d) Antitumor activity against lung metastasis induced by B16F10-Luc melanoma cells. Data are expressed as averages \pm s.e.m.; $n=5$; $* P<0.05$; $* * * P<0.001$. (e) Accumulation of micelles in lungs having B16F10-Luc melanoma cells $24 \mathrm{~h}$ after intravenous injection. Data are expressed as averages \pm s.e.m., $n=6$, ${ }^{*} P<0.005$. (f) Ex vivo fluorescent microscopy of lung tissues bearing B16F10-Luc metastasis $24 \mathrm{~h}$ after the injection of fluorescently labeled DACHPt/m or PBA-DACHPt/m (Alexa Fluor 647; pink) and quantification of fluorescent intensity in metastatic regions. Data are expressed as the averages \pm s.e.m., $n=3,{ }^{*} P<0.005$. Reproduced, with permission, from Deshayes et al. ${ }^{47}$ A full color version of this figure is available at Polymer Journal online.

specifically to tumor sites. ${ }^{15,46,47}$ For example, Crich et al. ${ }^{46}$ recently reported a gadolinium macrocyclic complex conjugated with PBA

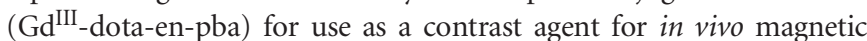
resonance imaging. A strong correlation between $\mathrm{Gd}^{\mathrm{III}}$-dota-en-pba uptake and the sialylation level in vitro, due to specific recognition of the overexpressed SA by the PBA-conjugated reporter, was first confirmed. Then, an in vivo experiment was carried out using a mouse model bearing a tumor xenograft (obtained by subcutaneous injection of B16-F10 cells), and the feasibility of SA-enhanced tumor imaging was demonstrated.

In a recent study, we described a PBA-modified (as a ligand to SA) polymeric micelle encapsulating anticancer drugs (Figure 3$)^{47}$ as a route to obtain tumor-specific chemotherapy. The polymeric micelles, which were self-assembled from biodegradable and biocompatible amphiphilic block copolymers, represent a promising platform for the delivery of anticancer drugs, as attested by the number of similar formulations currently in clinical trials. ${ }^{48}$ These nano-sized polymer micelles, typically several tens of nanometers in size, can effectively accumulate at the sites of tumors owing to their leaky vasculature and dysfunctional lymphatic drainage, an effect called enhanced permeability and retention (EPR effect). ${ }^{48,49}$ Ligand-mediated drug targeting is an attractive strategy for increasing the efficiency of chemotherapies. Indeed, SA has been targeted in vitro by using lectin ${ }^{50}$ and antibodies, ${ }^{51}$ such as the tumoral marker CA19-9, for the detection of Sialyl-Lewis ${ }^{\mathrm{a}}$ in gastrointestinal tumors. Nevertheless, these approaches have been difficult to translate to in vivo applications, mainly because of their immunogenicity. In this context, PBA-based SA targeting, which is non-toxic and nonimmunogenic, represents an attractive option. Moreover, because SA is universally present on biological surfaces, including red blood cells and the luminal surfaces of vascular endothelium, ${ }^{52,53}$ the ligand must be 'temporarily silenced' until reaching the site of tumors. Owing to its unique $\mathrm{pH}$-dependent binding modality, which is distinct from other competing sugars, the PBA-SA interaction can address this challenge. That is, the ligand can be designed to be shielded by other competing sugars while in the bloodstream ( $\mathrm{pH} 7.4$ ) and become increasingly SA-specific when exposed to the acidic conditions of the intratumoral environment. 

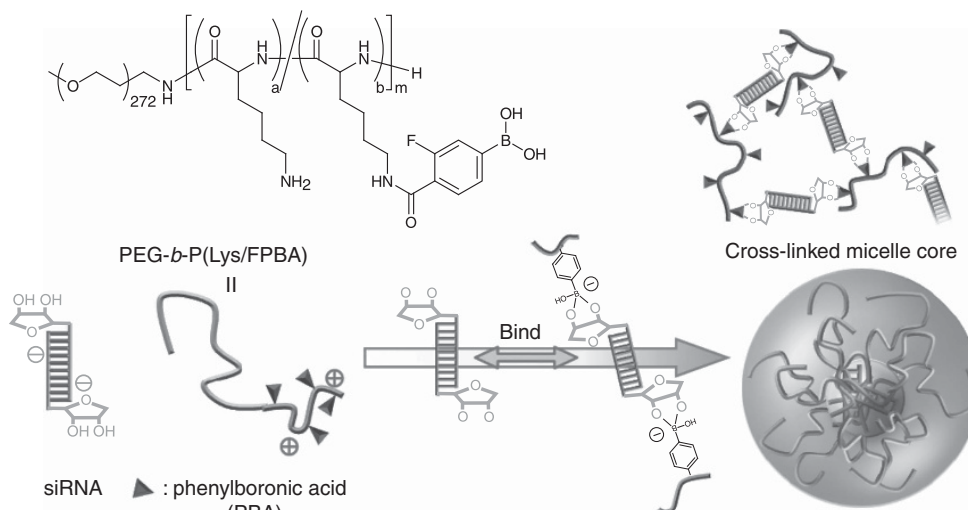

(PBA)
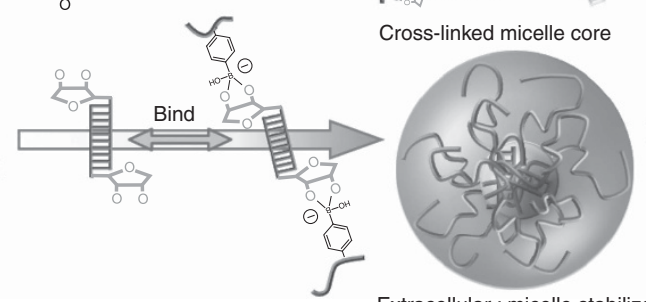

Extracellular : micelle stabilizaion

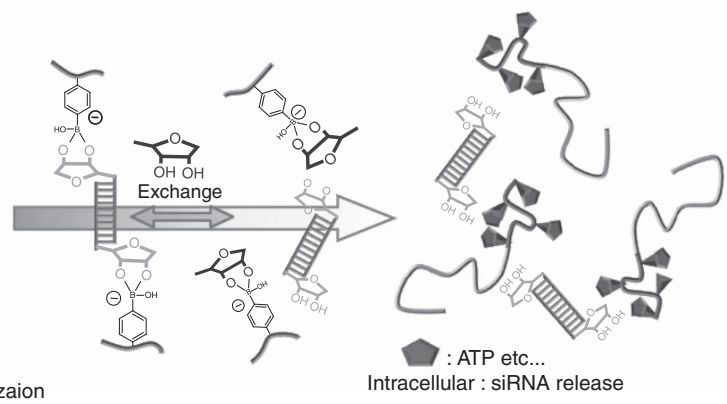

Figure 5 Schematic representation of the PBA-based strategy for siRNA delivery. The chemical formula of the polymer, enhanced stability of the micelle and mechanism of selective intracellular release are shown. Reproduced, with permission, from Naito et al. ${ }^{25} \mathrm{~A}$ full color version of this figure is available at Polymer Journal online.

a

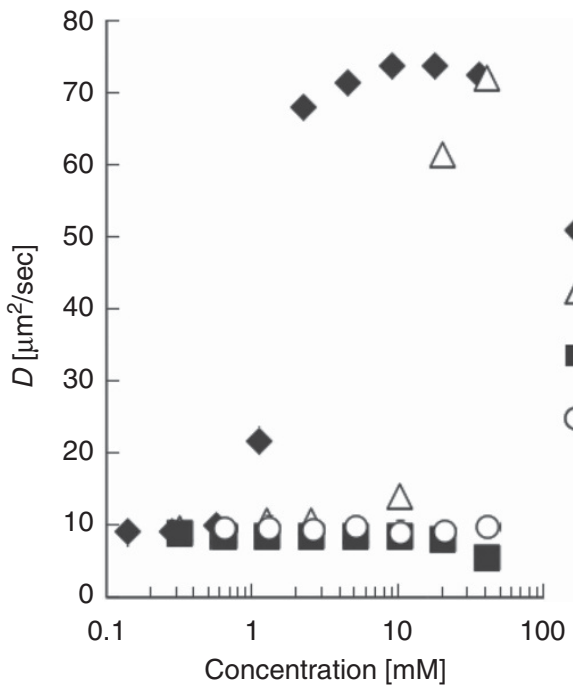

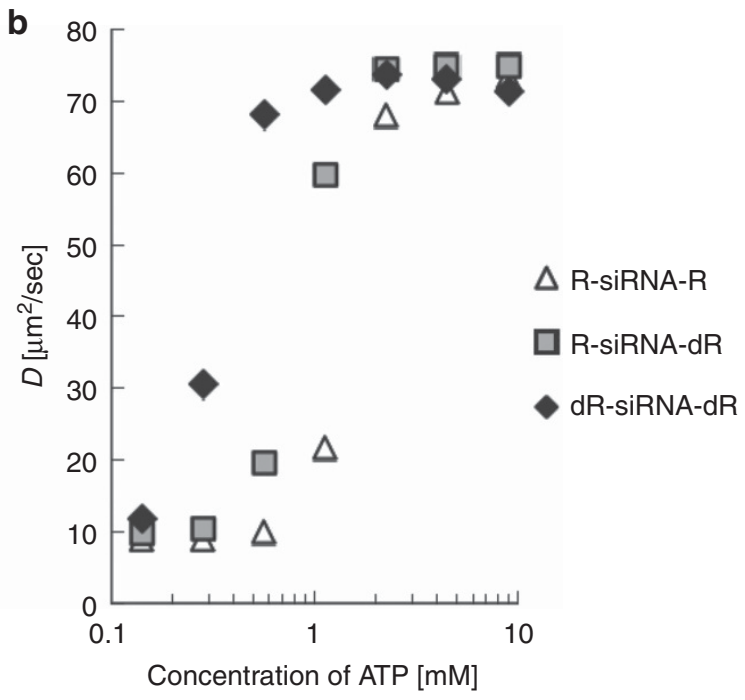

C

ATP

$\triangle$ UMP

dTMP

Glucose<smiles>Nc1ncnc2c1ncn2C1O[C@H](COP(=O)([O-])OP(=O)([O-])OP(=O)([O-])[O-])[C@@H](O)[C@H]1O</smiles>

ATP

UMP<smiles>Cc1cn(C2CC(O)C(OP(=O)([O-])[O-])O2)c(=O)[nH]c1=O</smiles>

dTMP<smiles>OCC1OC(O)[C@@H](O)[C@H](O)C1O</smiles>

Glucose

Figure 6 Ribose-specific stabilization of the complex and its sensitivity to a changing environment. (a) Changes in the diffusion coefficient of Cy3-labeled siRNAs complexed with PEG- $b-P(\text { Lys/FPBA } 23)_{42}$ as a function of various additive concentrations after a $1 \mathrm{~h}$ incubation. (b) Changes in the diffusion coefficient of siRNAs with different numbers of ribose functionalities when complexed with PEG- $b-\mathrm{P}\left(\mathrm{Lys} / \mathrm{FPBA} \mathrm{F}_{23}\right)_{42}$ as a function of ATP concentration. (c) Chemical formulas of the additives. Reproduced, with permission, from Naito et al. ${ }^{25}$

Micelles were prepared through coordination between an anticancer agent, (1,2-diaminocyclohexane)platinum(II) (DACHPt), and the carboxylic groups of PBA end-functionalized poly(ethylene glycol)- $b$-poly(L-glutamic acid) [PBA-PEG- $b$-PLGA] copolymers in an aqueous solution (Figure 3). The ability of the PBA-modified micelle (PBA-DACHPt/m) to bind SA epitopes in cancer cells was first studied in vitro by evaluating the cellular uptake and cytotoxicity of fluorescently labeled micelles using B16F10 cells. A significantly enhanced rate of cellular uptake of the PBA-modified micelle
(PBA-DACHPt $/ \mathrm{m}$ ) was confirmed compared with the control groups, which included non-PBA-modified micelles (DACHPt $/ \mathrm{m}$ ) and those blocked by the addition of free PBA and treated by sialidase (to cleave SA from the cells). Owing to its enhanced cellular uptake, the PBA-DACHPt $/ \mathrm{m}$ showed a significantly higher cytotoxicity than the control DACHPt $/ \mathrm{m}$. PBA-DACHPt $/ \mathrm{m}$ was further evaluated in vivo against orthotopic and metastatic tumor models. The orthotopic tumor model was prepared by intradermal inoculation of mice with B16F10 cells. Here, PBA-DACHPt $/ \mathrm{m}$ showed higher antitumor 
a

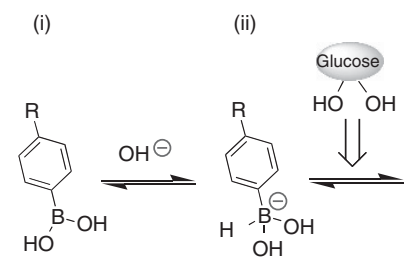

c

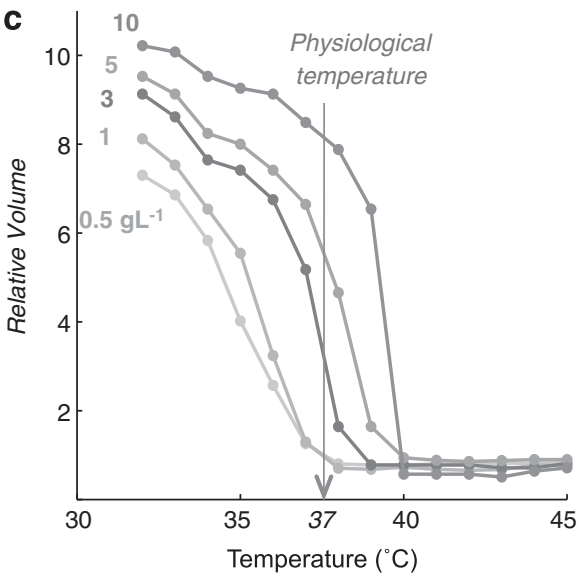

(iii)

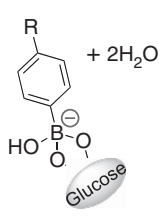

d

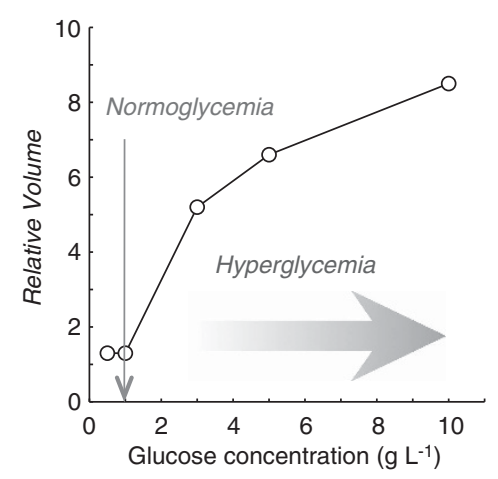

Figure 7 (a) Glucose-dependent equilibria of phenylboronic acid. (b) Structures of the monomers contained in the gel along with their optimized molar amounts in the feed solution for achieving glucose sensitivity at physiological pH and temperature ( $\mathrm{pH} 7.4$ and $37^{\circ} \mathrm{C}$ ). (c) Phase diagram of the gel showing the equilibrium volume changes as a function of temperature for various glucose concentrations investigated at $\mathrm{pH}$ 7.4. The green arrow indicates physiological temperature $\left(37^{\circ} \mathrm{C}\right)$. (d) Volume change of the gel as a function of the glucose concentration at $37^{\circ} \mathrm{C}$. The green arrow indicates the normoglycemic concentration of glucose $\left(1 \mathrm{gl}^{-1}\right)$. Reproduced, with permission, from Matsumoto et al..$^{73} \mathrm{~A}$ full color version of this figure is available at Polymer Journal online.

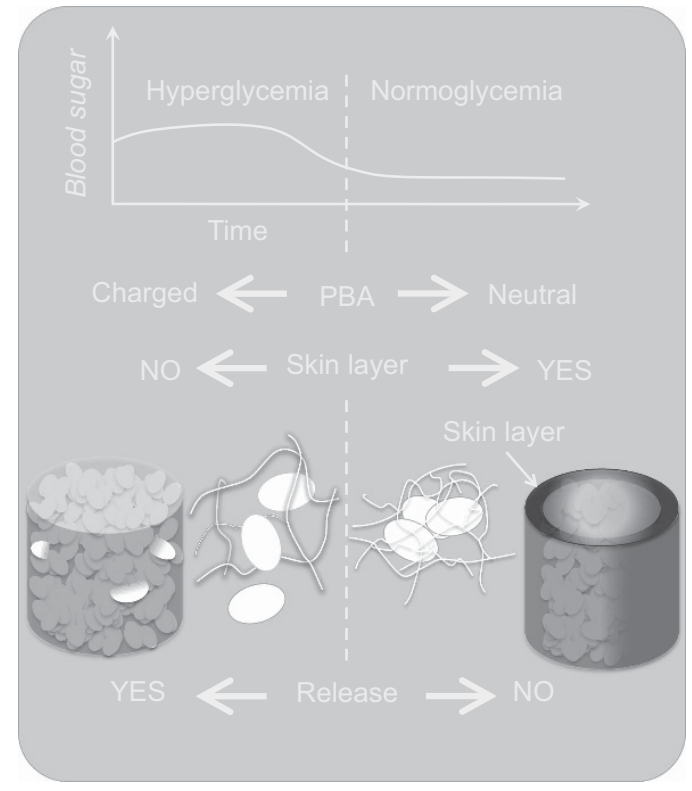

Scheme 2 Schematic illustration of self-regulated insulin delivery accomplished by a PBA-containing gel. A full color version of this scheme is available at Polymer Journal online. efficacy than DACHPt/m (Figure 4c), in line with the enhanced accumulation and retention at the tumor site (Figure 4b). PBA$\mathrm{DACHPt} / \mathrm{m}$ was further studied for its efficacy against bioluminescent lung metastasis, which was modeled by intravenous injection of B16F10 cells expressing luciferase (B16F10-Luc) in BALB/c nu/nu mice. While free oxaliplatin showed no antitumor effect (Figure $4 \mathrm{~d}$ ), both DACHPt $/ \mathrm{m}$ and PBA-DACHPt $/ \mathrm{m}$ significantly inhibited the progression of the metastasis. Moreover, PBA-DACHPt $/ \mathrm{m}$ again proved to be more effective than $\mathrm{DACHPt} / \mathrm{m}$, resulting in a twofold lower bioluminescent intensity (Figure $4 \mathrm{~d} ; P<0.05$ ). The enhanced activity of PBA-DACHPt $/ \mathrm{m}$ was consistent with the increased platinum accumulation of these micelles in metastatic lungs $24 \mathrm{~h}$ after injection (Figure 4e), and the specificity of PBA-DACHPt/m to the metastatic sites in the lungs was confirmed histologically (Figure 4f). Importantly, these enhancements in antitumor activity did not come at the expense of side effects; the body weight of the mice remained stable even after repeated administration of the micelles. These findings show feasibility for achieving enhanced drug delivery to the site of tumors via PBA-SA interactions.

\section{ENVIRONMENT-SENSITIVE SIRNA DELIVERY}

There is growing interest in the delivery of small interfering RNA (siRNA) due to its ability to silence genes in a highly sequence-specific manner. ${ }^{54,55}$ One major approach is to develop formulations based on polyion complex (PIC) micelles, which instantly form in an aqueous 

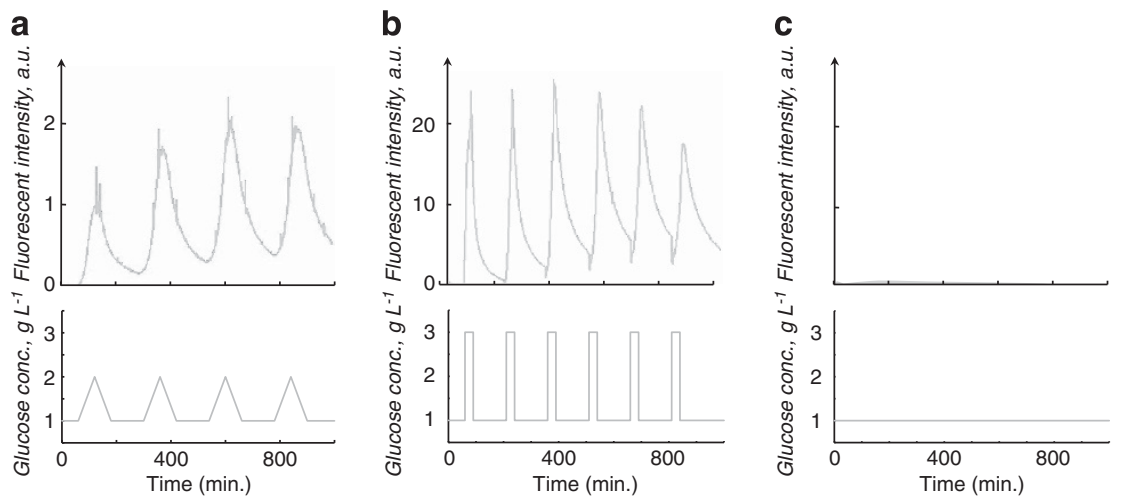

Figure 8 (Top) Time-course changes in the fluorescence intensity of FITC-labeled bovine insulin released from the gel at physiological conditions ( $\mathrm{pH}$ 7.4, $I=0.15,37^{\circ} \mathrm{C}$ ). (Bottom) Temporal patterns of the fluctuation in glucose concentration investigated in each (a-c) experiment. Reproduced, with permission, from Matsumoto et al. ${ }^{73} \mathrm{~A}$ full color version of this figure is available at Polymer Journal online.

environment, using electrostatic interactions between anionic siRNA and cationic polymers. ${ }^{56}$ An important issue is to stabilize the PIC micelles in the bloodstream to protect the siRNA from degradation by endogenous RNases, but in a way that allows destabilization (to release the siRNA) upon reaching the intracellular targets. Many attempts have been reported to date, the majority of which focus on either one or combinations of the following three approaches: covalent conjugation of siRNA to a homing polymer, ${ }^{57-61}$ introduction of hydrophobic moieties to reinforce core-aggregation ${ }^{62-64}$ and cross-linking core aggregates via disulfide bridging. ${ }^{65,66}$ However, these combination approaches often result in a highly complicated structure and method of preparation. To address this issue, PBA-ribose interactions provide a sophisticated solution.

Our strategy capitalizes solely on the PBA functionality, which incorporates all of the aforementioned modes of stabilization (Figure 5). ${ }^{25}$ First, the polymer pendent PBA groups can serve as sites for chemical conjugation with ribose of siRNA. Second, when electrostatically condensed to form PIC micelles, intermolecular cross-links become plausible because of the bis-bidentate ribose arrangement at each $3^{\prime}$-end of the double-stranded siRNA, thereby stabilizing the complex. Third, PBA is unique in that it undergoes a marked inversion in the level of hydrophobicity depending on the degree of acid disassociation, ${ }^{23}$ which is also dependent on the ribose (siRNA or other competing moieties) concentration in the milieu. These characteristics can be exploited to fine-tune and switch the stability of the complex, a feature relevant to achieving inter- and intracellular sensitivities.

Poly(ethylene glycol)-block-poly(L-lysine) (PEG- $b$-PLys), the lysine residues of which were quantitatively modified with 3-fluoro-4carboxyphenylboronic acid (FPBA) to varying degrees, was prepared to serve as a platform cationic polymer. The weight-average molecular weight $\left(M_{\mathrm{w}}\right)$ of PEG and the mean degree of polymerization of PLys were determined to be 12000 and 42, respectively. On the basis of systemic studies, the degree of PBA modification, as well as the mixing ratio between the polymer and siRNA, was optimized. It was demonstrated that the specific binding between the pendent PBA moieties and $3^{\prime}$-end ribose at both ends of the double-stranded siRNA and the hydrophobic interactions of PBA cooperatively contribute to the stability of the complex in quasi-extracellular conditions. We also found that the optimized complex is disrupted in response to adenosine triphosphate (ATP) at a range comparable to its intracellular concentration (Figure 6). Although still preliminary, the obtained PIC micelle showed the dose-dependent silencing capability of the polo-like kinase 1 (PLK-1) gene, a well-known proto-oncogene in the human renal carcinoma cell (OSRC-2) line, with minimal cytotoxicity. This PBA-assisted PIC micelle may have potential for intracellular environment-selective delivery of siRNA.

\section{SELF-REGULATED DELIVERY OF INSULIN}

Although diabetes is not an infectious disease, it has been recognized as a 'pandemic' because of its worldwide prevalence. ${ }^{67}$ The current palliative treatment for insulin-dependent diabetes mellitus (IDDM) relies almost entirely on self-administration of insulin injections by patients, which not only impinges on the quality of life of patients but also fails to precisely control the dose of insulin. The development of self-regulated insulin delivery systems has been an important topic of interest in material science. To this end, glucose oxidase and sugar-binding lectins, such as concanavalin A, have been used most frequently to endow homing polymer materials with glucose sensitivity. ${ }^{68}$ However, these protein-based materials are not compatible with long-term use and storage due to their denaturing and cytotoxic natures. We envision a PBA gel-based system as a completely synthetic alternative to these materials.

As illustrated in Figure 7a, PBA in water exists in equilibrium between uncharged (i) and anionically charged phenylboronate (ii) forms. Upon the addition of glucose, only the charged phenylboronate (ii) can form a stable complex with glucose (iii). Importantly, glucose binding results in a shift of the total equilibrium toward increased phenylboronate anions (ii and iii) and vice versa. This glucose-dependent change in the charged fraction of the phenylboronate, when coupled with an amphiphilic three-dimensional polymeric gel, gives rise to an abrupt and reversible change in the hydration of the gel ${ }^{69-73}$ due to changes in the counterions' osmotic pressure. In certain conditions, this reversible change in hydration can result in the formation of a surface-localized dehydrated layer, often called a 'skin layer,, ${ }^{71}$ which provides a means to instantly control the permeation of the preloaded insulin, as illustrated in Scheme 2. A great challenge, however, has been to achieve adequate glucose sensitivity at physiological $\mathrm{pH}$ and temperature (that is, $\mathrm{pH} 7.4$ and $37^{\circ} \mathrm{C}$ ) while also fine tuning the system to show a gated response to deviations in glucose concentration from normoglycemia (that is, $\sim 1 \mathrm{gl}^{-1}$ ). We have systemically explored structure-property correlations in (meth)acrylamide-based hydrogels, focusing on the control of the acidity of PBA and, concomitantly, the overall network hydrophilicity, ${ }^{23,70,72}$ and devised the optimized structure shown in Figure $7 \mathrm{~b} .^{73}$ 
Figures $8 \mathrm{a}-\mathrm{c}$ shows time-course profiles of insulin release from the gel under different patterns of glucose treatment, all investigated under physiological conditions. The release of the gel-loaded insulin was continuously controlled by the role of the skin layer in responding to the pattern of glucose. These results demonstrate that a non-protein-based, totally synthetic PBA gel can function to continuously control the provision of insulin under conditions relevant to human glucose homeostasis. Importantly, the nonequilibrium (skin layer-controlled) mechanism enables a remarkably shortened response time due to the short diffusion distance of the skin layer (typically on the order of $100 \mu \mathrm{m}$ ), which is an important criterion for the tight regulation of insulin dosage. Furthermore, in theory, this surface-limited mechanism allows for the prediction of the release profile, independent of the dimensions or topological structure of the gel. This feature may facilitate ease and accuracy in planning the administration of insulin. In addition, the 'dimensionindependency' may offer compatibility with other well-developed technologies, such as dwelling needles or semi-embedded devices. Aside from the skin layer-based strategy described herein, PBA gels could also be exploited as in other applications as mechanically functional elements, such as 'chemical valves' or 'pumps.' Regardless, this 'synthetic alternative' should offer a new material basis for the development of an 'artificial pancreas' with long-term stability and safety to treat diabetes.

\section{CONCLUSIONS}

This focus review highlighted some new aspects of PBA-based approaches to diagnostic and therapeutic applications that we are currently pursuing. Beyond traditional applications, such as glucose sensors and bio-separations, our efforts may indicate renewed potential for PBA-based polymer chemistry. Because of the inherent versatility of PBA chemistry along with its expanding library of derivatives (largely thanks to the advancement of Suzuki-Miyaura coupling), these new challenges may lead to additional prospects for their use as material platforms in future medical applications.

\section{CONFLICT OF INTEREST}

The authors declare no conflict of interest.

\section{ACKNOWLEDGEMENTS}

This study was supported by the Japan Science and Technology Agency (JST), Core Research of Evolutional Science \& Technology (CREST), and the Japan Society for the Promotion of Science (JSPS) through a grant-in-aid for young scientists (A) and, in part, by JSPS through the 'Funding Program for World-Leading Innovative R\&D on Science and Technology (FIRST Program), initiated by the Council for Science and Technology Policy (CSTP).

1 Lorand, J. P. \& Edwards, J. O. Polyol complexes and structure of the benzeneboronate ion. J. Org. Chem. 24, 769-774 (1959).

2 Foster, A. B. Zone electrophoresis of carbohydrates. Adv. Carbohydr. Chem. 12, 81-115 (1957).

3 Boeseken, J. The use of boric acid for the determination of the configuration of carbohydrates. Adv. Carbohydr. Chem. 4, 189-210 (1949).

4 Aronoff, S., Chen, T. C. \& Cheveldayoff, M. Complexation of D-glucose with borate Carbohydr. Res. 40, 299-309 (1975).

5 Gehrke, C. W., Kuo, K. C., Davis, G. E., Suits, R. D., Waalkes, T. P. \& Borek, E. Chromatography and modifications of nucleosides. J. Chromatogr. 150, 455-476 (1978).

6 Hase, S., Hatanaka, K., Ochiai, K. \& Shimizu, H. Improved method for the component sugar analysis of glycoproteins by pyridylamino sugars purified with immobilized boronic acid. Biosci. Biotech. Biochem. 56, 1676-1677 (1992).

7 Rosenberg, M., Wiebers, J. L. \& Gilham, P. T. Interactions of nucleotides, polynucleotides, and nucleic acids with dihydroxyboryl-substituted celluloses. Biochemistry 11 , 3623-3628 (1972)
8 Tuytten, R., Lemiere, F., Van Dongen, W., Witters, E., Esmans, E. L., Newton, R. P. \& Dudley, E. Development of an on-line SPE-LC-ESI-MS method for urinary nucleosides: hyphenation of aprotic boronic acid chromatography with hydrophilic interaction LC-ESI-MS. Anal. Chem. 80, 1263-1271 (2008).

9 Tuytten, R., Lemiere, F., Esmans, E. L., Herrebout, W. A., Van Der Veken, B. J., Maes, B. U. W., Witters, E., Newton, R. P. \& Dudley, E. Role of nitrogen lewis basicity in boronate affinity chromatography of nucleosides. Anal. Chem. 79, 6662-6669 (2007)

10 James, T. D., Sandanayake, K. R. A. S. \& Shinkai, S. Chiral discrimination of monosaccharides using a fluorescent molecular sensor. Nature 374, 345-347 (1994).

11 James, T. D., Sandanayake, K. R. A. S., Iguchi, R. \& Shinkai, S. Novel saccharidephotoinduced electron transfer sensors based on the interaction of boronic acid and amine. J. Am. Chem. Soc. 117, 8982-8987 (1995).

12 James, T. D., Sandanayake, K. R. A. S. \& Shinkai, S. Saccharide sensing with molecular receptors based on boronic acid. Angew. Chem. Int. Ed. Engl. 35, 1910-1922 (1996).

13 Kanayama, N. \& Kitano, H. Recognition of sugars by boronic acid-carrying self-assembled monolayer. Langmuir 16, 577-583 (2000).

14 Shoji, E. \& Freund, M. S. Potentiometric saccharide detection based on the $\mathrm{p} K$ changes of poly(aniline boronic acid). J. Am. Chem. Soc. 124, 12486-12493 (2002).

15 Ellis, G. A., Palte, M. J. \& Raines, R. T. Boronate-mediated biologic delivery. J. Am. Chem. Soc. 134, 3631-3634 (2012)

16 Westmark, P. R. \& Smith, B. D. Boronic acids facilitate the transport of ribonucleosides through lipid bilayers. J. Pharm. Sci. 85, 266-269 (1996).

17 Kumar, A., Hozo, I., Wheatley, K. \& Djulbegovic, B. Thalidomide versus bortezomib based regimens as first-line therapy for patients with multiple myeloma: a systematic review. Am. J. Hematol. 86, 18-24 (2011).

18 Melendez, M., Rosario, O., Zayas, B. \& Rodriguez, J. F. HPLC-MS/MS method for the intracellular determination of ribavirin monophosphate and ribavirin triphosphate in CEMss cells. J. Pharmaceut. Biomed. Anal. 49, 1233-1240 (2009).

19 Peng, Q., Chen, F., Zhong, Z. \& Zhuo, R. Enhanced gene transfection capability of polyethylenimine by incorporating boronic acid groups. Chem. Commun. 46 , 5888-5890 (2010).

20 Chen, X., Scgauder, S., Potier, N., Dorsselaer, A. V., Pelczer, I., Bassler, B. L. \& Hughson, F. M. Structural identification of a bacterial quorum-sensing signal containing boron. Nature 415, 545-549 (2002).

21 Pasparakis, G. Cockayne, A. \& Alexander, C. Control of bacterial aggregation by thermoresponsive glycopolymers. J. Am. Chem. Soc. 129, 11014-11015 (2007).

22 Uchimura, E., Otsuka, H., Okano, T., Sakurai, Y. \& Kataoka, K. Totally synthetic polymer with lectin-like function: induction of killer cells by the copolymer of 3-acrylamidophenylboronic acid with N, N-dimethylacrylamide. Biotechnol. Bioeng. 72, 307-314 (2001)

23 Matsumoto, A., Ikeda, S., Harada, A. \& Kataoka, K. Glucose-responsive polymer bearing a novel phenylborate derivative as a glucose-sensing moiety operating at physiological pH conditions. Biomacromolecules 4, 1410-1416 (2003).

24 Su, J., Chen, F., Cryns, V. L. \& Messersmith, P. B. Catechol polymers for ph-responsive targeted drug delivery to cancer cells. J. Am. Chem. Soc. 133, 11850-11853 (2011).

25 Naito, M., Ishii, T., Matsumoto, A., Miyata, K., Miyahara, Y. \& Kataoka, K. A phenylboronate-functionalized polyion complex micelle for ATP-triggered release of siRNA. Angew. Chem. Int. Ed. 51, 10751 (2012).

26 Fukuda, M. Possible roles of tumor-associated carbohydrate antigens. Cancer Res. 56, 2237-2244 (1996)

27 Bertozzi, C. R. \& Kiessling, L. L. Chemical glycobiology. Science 291, 2357-2364 (2001)

28 Raman, R., Raguram, S., Venkataraman, G., Paulson, J. C. \& Sasisekharan, R Glycomics: an integrated systems approach to structure-function relationships of glycans. Nat. Methods 2, 817-824 (2005).

29 Hakomori, S. Tumor malignancy defined by aberrant glycosylation and sphingo(glyco)lipid metabolism. Cancer Res. 56, 5309-5318 (1996).

30 Dube, D. H. \& Bertozzi, C. R. Glycans in cancer and inflammation-potential for therapeutics and diagnostics. Nat. Rev. Drug Discov. 4, 477-488 (2006)

31 Xu, Y., Sette, A., Sidney, J., Gendler, S. J. \& Franco, A. Special feature: glycobiology of xenotransplantation and cancer part I. Immunol. Cell Biol. 83, 440-448 (2005).

32 Kannagi, R., Sakuma, K., Miyazaki, K., Lim, K. T., Yusa, A., Yin, J. \& Izawa, M Altered expression of glycan genes in cancers induced by epigenetic silencing and tumor hypoxia: clues in the ongoing search for new tumor markers. Cancer Sci. 101, 586-593 (2010)

33 Kannagi, R., Izawa, M., Koike, T., Miyazaki, K. \& Kimura, N. Carbohydrate-mediated cell adhesion in cancer metastasis and angiogenesis. Cancer Sci. 95, 377-384 (2004).

34 Otsuka, H., Uchimura, E., Koshino, H., Okano, T. \& Kataoka, K. Anomalous binding profile of phenylboronic acid with $\mathrm{N}$-acetylneuraminic acid (Neu5Ac) in aqueous solution with varying pH. J. Am. Chem. Soc. 125, 3493-3502 (2003).

35 Djanashvili, K., Frullano, L. \& Peters, J. A. Molecular recognition of sialic acid end groups by phenylboronates. Chem.-Eur. J. 11, 4010-4018 (2005).

36 Matsumoto, A. Sato, Kataoka, N. \& Miyahara, K. Y. Noninvasive sialic acid detection at cell membrane by using phenylboronic acid modified self-assembled monolayer gold electrode. J. Am. Chem. Soc. 131, 12022-12024 (2009).

37 Matsumoto, A., Cabral, H., Sato, N., Kataoka, K. \& Miyahara, Y. Assessment of tumor metastasis by the direct determination of cell-membrane sialic acid expression. Angew. Chem. Int. Ed. 49, 5494-5497 (2010).

38 Matsumoto, A. \& Miyahara, Y. Current and emerging challenges in field effect transistor based bio-sensing. Nanoscale 5, 10702-10718 (2013). 
39 Chari, S. N. \& Nath, N. Sialic acid content and sialidase activity of polymorphonuclear leucocytes in diabetes mellitus. Am. J. Med. Sci. 288, 18-20 (1984).

40 Rogers, M. E., Williams, D. T., Niththyananthan, R., Rampling, M. W., Heslop, K. E. \& Johnston, D. G. Decrease in erythrocyte glycophorin sialic acid content is associated with increased erythrocyte aggregation in human diabetes. Clin. Sci. 82, 309-313 (1992).

41 Vahalkar, G. S. \& Haldankar, V. A. RBC membrane composition in insulin dependent diabetes mellitus in context of oxidative stress. Ind. J. Clin. Biochem. 23, 223-226 (2008).

42 Fidler, I. J. Selection of successive tumour lines for metastasis. Nat. New Biol. 242, 148-149 (1973).

43 Parhar, R. S. \& Lala, P. K. Amelioration of B16F10 melanoma lung metastasis in mice by a combination therapy with indomethacin and interleukin 2. J. Exp. Med. 165 , 14-28 (1987).

44 Edinger, M., Cao, Y. A., Hornig, Y. S., Jenkins, D. E., Verneris, M. R., Bachmann, M. H., Negrin, R. S. \& Contag, C. H. Advancing animal models of neoplasia through in vivo bioluminescence imaging. Eur. J. Cancer 38, 2128-2136 (2002).

45 Han, E., Ding, L. \& Ju, H. Highly sensitive fluorescent analysis of dynamic glycan expression on living cells using glyconanoparticles and functionalized quantum dots. Anal. Chem. 83, 7006-7012 (2011).

46 Crich, S. G., Alberti, D., Szabo, I., Aime, S. \& Djanashvili, K. MRI visualization of melanoma cells by targeting overexpressed sialic acid with a Gd ${ }^{\prime \prime \prime}$-dota-en-pba imaging reporter. Angew. Chem. Int. Ed. 52, 1161 (2013).

47 Deshayes, S., Cabral, H., Ishii, T., Miura, Y., Kobayashi, S., Yamashita, T., Matsumoto, A., Miyahara, Y., Nishiyama, N. \& Kataoka, K. Phenylboronic acid-installed polymeric micelles for targeting sialylated epitopes in solid tumors. J. Am. Chem. Soc. 135, 15501-15507 (2013).

48 Matsumura, Y. \& Kataoka, K. Preclinical and clinical studies of anticancer agentincorporating polymer micelles. Cancer Sci. 100, 572-579 (2009).

49 Matsumura, Y. \& Maeda, H. A new concept for macromolecular therapeutics in cancer chemotherapy: mechanism of tumoritropic accumulation of proteins and the antitumor agent smancs. Cancer Res. 46, 6387-6392 (1986).

50 Sharon, N. Lectins: carbohydrate-specific reagents and biological recognition molecules. J. Biol. Chem. 282, 2753-2764 (2007)

51 Koprowski, H., Herlyn, M., Steplewski, Z. \& Sears, H. F. Specific antigen in serum of patients with colon carcinoma. Science 212, 53-55 (1981).

52 Durocher, J. R., Payne, R. C. \& Conrad, M. E. Role of sialic acid in erythrocyte survival. Blood 45, 11-20 (1975).

53 Born, G. V. \& Palinski, W. Unusually high concentrations of sialic acids on the surface of vascular endothelia. Br. J. Exp. Pathol. 66, 543-549 (1985).

54 Elbashir, S. M., Harborth, J., Lendeckel, W., Yalcin, A., Weber, K. \& Tuschl, T. Duplexes of 21-nucleotide RNAs mediate RNA interference in cultured mammalian cells. Nature 411, 494-498 (2001).

55 Whitehead, K. A., Langer, R. \& Anderson, D. G. Knocking down barriers: advances in siRNA delivery. Nat. Rev. Drug Discov. 8, 129-138 (2009).

56 Nishiyama, N. \& Kataoka, K. Current state, achievements, and future prospects of polymeric micelles as nanocarriers for drug and gene delivery. Pharmacol. Ther. 112, 630-648 (2006).

57 Oishi, M., Nagasaki, Y., Itaka, K., Nishiyama, N. \& Kataoka, K. Lactosylated poly(ethylene glycol)-siRNA conjugate through acid-labile beta-Thiopropionate linkage to construct $\mathrm{pH}$-sensitive polyion complex micelles achieving enhanced gene silencing in hepatoma cells. J. Am. Chem. Soc. 127, 1624-1625 (2005).
58 Rozema, D. B., Lewis, D. L., Wakefield, D. H., Wong, S. C., Klein, J. J., Roesch, P. L., Bertin, S. L., Reppen, T. W., Chu, Q., Blokhin, A. V., Hagstrom, J. E. \& Wolf, J. A. Dynamic polyconjugates for targeted in vivo delivery of siRNA to hepatocytes. Proc. Natl Acad. Sci. USA 104, 12982-12987 (2007).

59 Meyer, M., Dohmen, C., Philipp, A., Kiener, D., Maiwald, G., Scheu, C., Ogris, M. \& Wagner, E. Synthesis and biological evaluation of a bioresponsive and endosomolytic siRNA - polymer conjugate. Mol. Pharm. 6, 752-762 (2009).

60 Mok, H., Lee, S. H., Park, J. W. \& Park, T. G. Multimeric small interfering ribonucleic acid for highly efficient sequence-specific gene silencing. Nat. Mater. 9, 272-278 (2010).

61 Takemoto, H., Ishii, A., Miyata, K., Nakanishi, M., Oba, M., Ishii, T., Yamasaki, Y., Nishiyama, N. \& Kataoka, K. Polyion complex stability and gene silencing efficiency with a siRNA-grafted polymer delivery system. Biomaterials 31, 8097-8105 (2010).

62 Alshamsan, A., Haddadi, A., Incani, V., Samuel, J., Lavasanifar, A. \& Uludag, H. Formulation and delivery of siRNA by oleic acid and stearic acid modified polyethylenimine. Mol. Pharm. 6, 121-133 (2009).

63 Kim, W. J., Christensen, L. V., Jo, S., Yockman, J. W., Jeong, J. H., Kim, Y. H. \& Kim, S. W. Cholesteryl oligoarginine delivering vascular endothelial growth factor siRNA effectively inhibits tumor growth in colon adenocarcinoma. Mol. Ther. 14, 343-350 (2006).

64 Kim, H. J., Ishii, A., Miyata, K., Lee, Y., Wu, S., Oba, M., Nishiyama, N. \& Kataoka, K. Introduction of stearoyl moieties into a biocompatible cationic polyaspartamide derivative, PAsp(DET), with endosomal escaping function for enhanced siRNAmediated gene knockdown. J. Control. Res. 145, 141-148 (2010).

65 Matsumoto, S., Christie, R. J., Nishiyama, N., Miyata, K., Ishii, A., Oba, M., Koyama, H., Yamasaki, Y. \& Kataoka, K. Environment-responsive block copolymer micelles with a disulfide cross-linked core for enhanced siRNA delivery. Biomacromolecules 10, 119-127 (2009).

66 Christie, R. J., Miyata, K., Matsumoto, Y., Nomoto, T., Menasco, D., Lai, T. C., Pennisi, M., Osada, K., Fukushima, S., Nishiyama, N., Yamasaki, Y. \& Kataoka, K. Effect of polymer structure on micelles formed between siRNA and cationic block copolymer comprising thiols and amidines. Biomacromolecules 12, 3174-3185 (2011).

67 United Nations. Resolution 61/225: World Diabetes Day from 83rd plenary meeting, 20 December (2006)

68 Miyata, T., Uragami, T. \& Nakamae, K. Biomolecule-sensitive hydrogels. Adv. Drug Deliv. Rev. 54, 79 (2002).

69 Kataoka, K., Miyazaki, H., Bunya, M., Okano, T. \& Sakurai, Y. Totally synthetic polymer gels responding to external glucose concentration: Their preparation and application to on-off regulation of insulin release. J. Am. Chem. Soc. 120, 12694-12695 (1998).

70 Matsumoto, A., Yoshida, R. \& Kataoka, K. Glucose-responsive polymer gel bearing phenylborate derivative as a glucose-sensing moiety operating at the physiological $\mathrm{pH}$. Biomacromolecules 5, 1038-1045 (2004).

71 Matsumoto, A., Kurata, T., Shiino, D. \& Kataoka, K. Swelling and shrinking kinetics of totally synthetic, glucose-responsive polymer gel bearing phenylborate derivative as a glucose-sensing moiety. Macromolecules 37, 1502-1510 (2004).

72 Matsumoto, A., Yamamoto, K., Yoshida, R., Kataoka, K., Aoyagi, T. \& Miyahara, Y. A totally synthetic glucose responsive gel operating in physiological aqueous conditions. Chem. Commun. 46, 2203-2205 (2010).

73 Matsumoto, A., Ishii, T., Nishida, J., Matsumoto, H., Kataoka, K. \& Miyahara, Y. A synthetic approach toward self-regulated insulin delivery system. Angew. Chem., Int. Ed. 51, 2124 (2012). 